\title{
Optimal bounds for the Neuman-Sándor mean in terms of the first Seiffert and quadratic means
}

\author{
Wei-Ming Gong ${ }^{1}$, Xu-Hui Shen ${ }^{2}$ and Yu-Ming Chu*
}

\section{"Correspondence:}

chuyuming2005@126.com

'School of Mathematics and

Computation Science, Hunan City

University, Yiyang, 413000, China

Full list of author information is

available at the end of the article

\begin{abstract}
In this paper, we find the least value $\alpha$ and the greatest value $\beta$ such that the double inequality

$$
P^{\alpha}(a, b) Q^{1-\alpha}(a, b)<M(a, b)<P^{\beta}(a, b) Q^{1-\beta}(a, b)
$$

holds true for all $a, b>0$ with $a \neq b$, where $P(a, b), M(a, b)$ and $Q(a, b)$ are the first Seiffert, Neuman-Sándor and quadratic means of $a$ and $b$, respectively.
\end{abstract}

MSC: $26 \mathrm{E} 60$

Keywords: Neuman-Sándor mean; first Seiffert mean; quadratic mean

\section{Introduction}

Let $u, v$ and $w$ be the bivariate means such that $u(a, b)<w(a, b)<v(a, b)$ for all $a, b>0$ with $a \neq b$. The problems of finding the best possible parameters $\alpha$ and $\beta$ such that the inequalities $\alpha u(a, b)+(1-\alpha) v(a, b)<w(a, b)<\beta u(a, b)+(1-\beta) v(a, b)$ and $u^{\alpha}(a, b) v^{1-\alpha}(a, b)<$ $w(a, b)<u^{\beta}(a, b) v^{1-\beta}(a, b)$ hold for all $a, b>0$ with $a \neq b$ have attracted the interest of many mathematicians.

For $a, b>0$ with $a \neq b$, the first Seiffert mean $P(a, b)$ [1], the Neuman-Sándor mean $M(a, b)$ [2], the quadratic mean $Q(a, b)$ are defined by

$$
\begin{aligned}
& P(a, b)=\frac{a-b}{4 \arctan (\sqrt{a / b})-\pi}, \quad M(a, b)=\frac{a-b}{2 \sinh ^{-1}\left(\frac{a-b}{a+b}\right)}, \\
& Q(a, b)=\sqrt{\frac{a^{2}+b^{2}}{2}}
\end{aligned}
$$

respectively. In here, $\sinh ^{-1}(x)=\log \left(x+\sqrt{x^{2}+1}\right)$ is the inverse hyperbolic sine function.

Recently, the means $P, M$ and $Q$ have been the subject of intensive research. In particular, many remarkable inequalities for these means can be found in the literature [3-14]. The first Seiffert mean $P(a, b)$ can be rewritten as (see [2, Eq. (2.4)])

$$
P(a, b)=\frac{a-b}{2 \arcsin [(a-b) /(a+b)]} .
$$

\section{Springer}

○2013 Gong et al.; licensee Springer. This is an Open Access article distributed under the terms of the Creative Commons Attribution License (http://creativecommons.org/licenses/by/2.0), which permits unrestricted use, distribution, and reproduction in any medium, provided the original work is properly cited. 
Let $H(a, b)=2 a b /(a+b), L(a, b)=(b-a) /(\log b-\log a), A(a, b)=(a+b) / 2, T(a, b)=$ $(a-b) /[2 \arctan ((a-b) /(a+b))]$ and $C(a, b)=\left(a^{2}+b^{2}\right) /(a+b)$ be the harmonic, logarithmic, arithmetic, second Seiffert and contra-harmonic means of $a$ and $b$, respectively. Then it is known that the inequalities

$$
H(a, b)<L(a, b)<P(a, b)<A(a, b)<M(a, b)<T(a, b)<Q(a, b)<C(a, b)
$$

hold for all $a, b>0$ with $a \neq b$.

Neuman and Sándor $[2,15]$ proved that the inequalities

$$
\begin{aligned}
& \frac{\pi}{4 \log (1+\sqrt{2})} T(a, b)<M(a, b)<\frac{A(a, b)}{\log (1+\sqrt{2})}, \\
& \sqrt{2 T^{2}(a, b)-Q^{2}(a, b)}<M(a, b)<\frac{T^{2}(a, b)}{Q(a, b)}, \\
& H(T(a, b), A(a, b))<M(a, b)<L(A(a, b), Q(a, b)), \quad T(a, b)>H(M(a, b), Q(a, b)), \\
& M(a, b)<\frac{A^{2}(a, b)}{P(a, b)}, \quad A^{2 / 3}(a, b) Q^{1 / 3}(a, b)<M(a, b)<\frac{2 A(a, b)+Q(a, b)}{3}, \\
& \sqrt{A(a, b) T(a, b)}<M(a, b)<\sqrt{A^{2}(a, b)+T^{2}(a, b)}, \\
& \frac{A(x, y)}{A(1-x, 1-y)}<\frac{M(x, y)}{M(1-x, 1-y)}<\frac{T(x, y)}{T(1-x, 1-y)}, \\
& \frac{1}{A(1-x, 1-y)}-\frac{1}{A(x, y)}<\frac{1}{M(1-x, 1-y)}-\frac{1}{M(x, y)}<\frac{1}{T(1-x, 1-y)}-\frac{1}{T(x, y)}, \\
& A(x, y) A(1-x, 1-y)<M(x, y) M(1-x, 1-y)<T(x, y) T(1-x, 1-y)
\end{aligned}
$$

hold for all $a, b>0$ and $x, y \in(0,1 / 2]$ with $a \neq b$ and $x \neq y$.

Li et al. [16] proved that the double inequality $L_{p_{0}}(a, b)<M(a, b)<L_{2}(a, b)$ holds for all $a, b>0$ with $a \neq b$, where $L_{p}(a, b)=\left[\left(b^{p+1}-a^{p+1}\right) /((p+1)(b-a))\right]^{1 / p}(p \neq-1,0), L_{0}(a, b)=$ $1 / e\left(b^{b} / a^{a}\right)^{1 /(b-a)}$ and $L_{-1}(a, b)=(b-a) /(\log b-\log a)$ is the $p$ th generalized logarithmic mean of $a$ and $b$, and $p_{0}=1.843 \ldots$ is the unique solution of the equation $(p+1)^{1 / p}=$ $2 \log (1+\sqrt{2})$.

In [13], Neuman proved that the double inequalities

$$
Q^{\alpha}(a, b) A^{1-\alpha}(a, b)<M(a, b)<Q^{\beta}(a, b) A^{1-\beta}(a, b)
$$

and

$$
C^{\lambda}(a, b) A^{1-\lambda}(a, b)<M(a, b)<C^{\mu}(a, b) A^{1-\mu}(a, b)
$$

hold for all $a, b>0$ with $a \neq b$ if $\alpha \leq 1 / 3, \beta \geq 2[\log (2+\sqrt{2})-\log 3] / \log 2, \lambda \leq 1 / 6$ and $\mu \geq[\log (2+\sqrt{2})-\log 3] / \log 2$.

Jiang and Qi $[17,18]$ gave the best possible parameters $\alpha, \beta, t_{1}$ and $t_{2}$ in $(0,1 / 2)$ such that the inequalities

$$
\begin{aligned}
& Q(\alpha a+(1-\alpha) b, \alpha b+(1-\alpha) a)<M(a, b)<Q(\beta a+(1-\beta) b, \beta b+(1-\beta) a), \\
& Q_{t_{1}, p}(a, b)<M(a, b)<Q_{t_{2}, p}(a, b)
\end{aligned}
$$


hold for all $a, b>0$ with $a \neq b$ and $p \geq 1 / 2$, where $Q_{t, p}(a, b)=C^{p}(t a+(1-t) b, t b+(1-$ t)a) $A^{1-p}(a, b)$.

Inspired by inequalities (1.3) and (1.4), in this paper, we present the optimal upper and lower bounds for the Neuman-Sándor mean $M(a, b)$ in terms of the geometric convex combinations of the first Seiffert mean $P(a, b)$ and the quadratic mean $Q(a, b)$. All numerical computations are carried out using Mathematica software.

\section{Lemmas}

In order to establish our main result, we need several lemmas, which we present in this section.

Lemma 2.1 The double inequality

$$
x+\frac{x^{3}}{3}-\frac{2 x^{5}}{15}<\sqrt{1+x^{2}} \sinh ^{-1}(x)<x+\frac{x^{3}}{3}-\frac{2 x^{5}}{15}+\frac{8 x^{7}}{105}
$$

holds for $x \in(0,1)$.

Proof To show inequality (2.1), it suffices to prove that

$$
\omega_{1}(x)=\sqrt{1+x^{2}} \sinh ^{-1}(x)-\left(x+\frac{x^{3}}{3}-\frac{2 x^{5}}{15}\right)>0
$$

and

$$
\omega_{2}(x)=\sqrt{1+x^{2}} \sinh ^{-1}(x)-\left(x+\frac{x^{3}}{3}-\frac{2 x^{5}}{15}+\frac{8 x^{7}}{105}\right)<0
$$

for $x \in(0,1)$.

From the expressions of $\omega_{1}(x)$ and $\omega_{2}(x)$, we get

$$
\begin{aligned}
& \omega_{1}(0)=\omega_{2}(0)=0, \\
& \omega_{1}^{\prime}(x)=\frac{x \omega_{1}^{*}(x)}{\sqrt{1+x^{2}}}, \quad \omega_{2}^{\prime}(x)=\frac{x \omega_{2}^{*}(x)}{\sqrt{1+x^{2}}},
\end{aligned}
$$

where

$$
\begin{aligned}
& \omega_{1}^{*}(x)=\sinh ^{-1}(x)-\left(x-\frac{2 x^{3}}{3}\right) \sqrt{1+x^{2}}, \\
& \omega_{2}^{*}(x)=\sinh ^{-1}(x)-\left(x-\frac{2 x^{3}}{3}+\frac{8 x^{5}}{15}\right) \sqrt{1+x^{2}}, \\
& \omega_{1}^{*}(0)=\omega_{2}^{*}(0)=0, \\
& \omega_{1}^{* \prime}(x)=\frac{8 x^{4}}{3 \sqrt{1+x^{2}}}>0
\end{aligned}
$$

and

$$
\omega_{2}^{* \prime}(x)=-\frac{16 x^{6}}{5 \sqrt{1+x^{2}}}<0
$$

for $x \in(0,1)$. 
Therefore, inequality (2.2) follows from (2.4)-(2.7), and inequality (2.3) follows from (2.4)-(2.6) and (2.8).

Lemma 2.2 The inequality

$$
\frac{x^{3}}{\sqrt{1+x^{2}}}>\left[\sinh ^{-1}(x)\right]^{3}
$$

holds for $x \in(0,1)$.

Proof Let $x \in(0,1)$, then from (1.3) we have

$$
M(1+x, 1-x)>A^{2 / 3}(1+x, 1-x) Q^{1 / 3}(1+x, 1-x) .
$$

Therefore, Lemma 2.2 follows from (2.9).

\section{Lemma 2.3 The inequality}

$$
\sqrt{1-x^{2}} \arcsin (x)>x-\frac{x^{3}}{3}-\frac{x^{5}}{3}
$$

holds for $x \in(0,0.7)$, and the inequality

$$
\sqrt{1-x^{2}} \arcsin (x)<x-\frac{x^{3}}{3}-\frac{2 x^{5}}{15}
$$

holds for $x \in(0,1)$, where $\arcsin (x)$ is the inverse sine function.

Proof Let

$$
\begin{aligned}
& \varphi_{1}(x)=\sqrt{1-x^{2}} \arcsin (x)-x+\frac{x^{3}}{3}+\frac{x^{5}}{3}, \\
& \varphi_{2}(x)=\sqrt{1-x^{2}} \arcsin (x)-x+\frac{x^{3}}{3}+\frac{2 x^{5}}{15} .
\end{aligned}
$$

Then simple computations lead to

$$
\begin{aligned}
& \varphi_{1}(0)=\varphi_{2}(0)=0, \\
& \varphi_{1}^{\prime}(x)=\frac{x \varphi_{1}^{*}(x)}{\sqrt{1-x^{2}}}, \quad \varphi_{2}^{\prime}(x)=\frac{x \varphi_{2}^{*}(x)}{\sqrt{1-x^{2}}},
\end{aligned}
$$

where

$$
\begin{aligned}
& \varphi_{1}^{*}(x)=\left(x+\frac{5 x^{3}}{3}\right) \sqrt{1-x^{2}}-\arcsin (x), \\
& \varphi_{2}^{*}(x)=\left(x+\frac{2 x^{3}}{3}\right) \sqrt{1-x^{2}}-\arcsin (x) .
\end{aligned}
$$

Note that

$$
\varphi_{1}^{*}(0)=\varphi_{2}^{*}(0)=0, \quad \varphi_{1}^{*}(0.7)=0.1327 \ldots,
$$




$$
\begin{aligned}
& \varphi_{1}^{* \prime}(x)=\frac{x^{2}\left(9-20 x^{2}\right)}{3 \sqrt{1-x^{2}}}, \\
& \varphi_{2}^{*^{\prime}}(x)=-\frac{8 x^{4}}{3 \sqrt{1-x^{2}}}<0
\end{aligned}
$$

for $x \in(0,1)$.

From (2.17) we clearly see that $\varphi_{1}^{*}(x)$ is strictly increasing on $(0,3 \sqrt{5} / 10]$ and strictly decreasing on $[3 \sqrt{5} / 10,0.7)$. This in conjunction with (2.16) implies that

$$
\varphi_{1}^{*}(x)>0
$$

for $x \in(0,0.7)$.

Therefore, inequality (2.10) follows from (2.12), (2.14), (2.15) and (2.19), and inequality (2.11) follows from (2.12) and (2.14)-(2.16) together with (2.18).

Lemma 2.4 Let

$$
\Phi(x)=\frac{1}{\sqrt{1+x^{2}} \sinh ^{-1}(x)}-\frac{1}{x\left(1+x^{2}\right)} .
$$

Then the inequality

$$
\Phi(x)>\frac{2 x}{3}-\frac{34 x^{3}}{45}+\frac{754 x^{5}}{945}-x^{7}
$$

holds for $x \in(0,0.7)$, and

$$
\Phi(x)<\frac{2 x}{3}-\frac{34 x^{3}}{45}+\frac{4 x^{5}}{5}
$$

holds for $x \in(0,1)$.

Proof To show inequalities (2.20) and (2.21), it suffices to prove that

$$
\begin{aligned}
\phi_{1}(x):= & x\left(1+x^{2}\right) \sinh ^{-1}(x)\left[\Phi(x)-\left(\frac{2 x}{3}-\frac{34 x^{3}}{45}+\frac{754 x^{5}}{945}-x^{7}\right)\right] \\
= & x \sqrt{1+x^{2}}-\sinh ^{-1}(x) \\
& -x\left(1+x^{2}\right) \sinh ^{-1}(x)\left(\frac{2 x}{3}-\frac{34 x^{3}}{45}+\frac{754 x^{5}}{945}-x^{7}\right)>0
\end{aligned}
$$

for $x \in(0,0.7)$, and

$$
\begin{aligned}
\phi_{2}(x):= & x\left(1+x^{2}\right) \sinh ^{-1}(x)\left[\Phi(x)-\left(\frac{2 x}{3}-\frac{34 x^{3}}{45}+\frac{4 x^{5}}{5}\right)\right] \\
= & x \sqrt{1+x^{2}}-\sinh ^{-1}(x) \\
& -x\left(1+x^{2}\right) \sinh ^{-1}(x)\left(\frac{2 x}{3}-\frac{34 x^{3}}{45}+\frac{4 x^{5}}{5}\right)<0
\end{aligned}
$$

for $x \in(0,1)$. 
From the expressions of $\phi_{1}(x)$ and $\phi_{2}(x)$, one has

$$
\begin{aligned}
& \phi_{1}(0)=\phi_{2}(0)=0, \\
& \phi_{1}^{\prime}(x)=\frac{x}{945 \sqrt{1+x^{2}}} \phi_{1}^{*}(x), \quad \phi_{2}^{\prime}(x)=-\frac{2 x}{45 \sqrt{1+x^{2}}} \phi_{2}^{*}(x),
\end{aligned}
$$

where

$$
\begin{aligned}
\phi_{1}^{*}(x)= & x\left(1,260+84 x^{2}-40 x^{4}+191 x^{6}+945 x^{8}\right) \\
& -2\left(630-168 x^{2}+120 x^{4}-764 x^{6}-4,725 x^{8}\right) \sqrt{1+x^{2}} \sinh ^{-1}(x), \\
\phi_{2}^{*}(x)= & x\left(18 x^{6}+x^{4}-2 x^{2}-30\right) \\
& +2\left(15-4 x^{2}+3 x^{4}+72 x^{6}\right) \sqrt{1+x^{2}} \sinh ^{-1}(x) .
\end{aligned}
$$

Note that

$$
\begin{aligned}
& 630-168 x^{2}+120 x^{4}-764 x^{6}-4,725 x^{8} \\
& >630-168 \times(0.7)^{2}-764 \times(0.7)^{6}-4,725 \times(0.7)^{8}=185.4 \ldots>0
\end{aligned}
$$

for $x \in(0,0.7)$.

Lemma 2.1 and equations (2.26)-(2.28) lead to

$$
\begin{aligned}
\phi_{1}^{*}(x)> & x\left(1,260+84 x^{2}-40 x^{4}+191 x^{6}+945 x^{8}\right) \\
& -2\left(630-168 x^{2}+120 x^{4}-764 x^{6}-4,725 x^{8}\right)\left(x+\frac{x^{3}}{3}-\frac{2 x^{5}}{15}+\frac{8 x^{7}}{105}\right) \\
= & \frac{x^{7}}{105}\left(157,311+1,151,003 x^{2}+307,438 x^{4}-120,076 x^{6}+75,600 x^{8}\right)>0
\end{aligned}
$$

for $x \in(0,0.7)$, and

$$
\begin{aligned}
\phi_{2}^{*}(x)> & x\left(18 x^{6}+x^{4}-2 x^{2}-30\right) \\
& +2\left(15-4 x^{2}+3 x^{4}+72 x^{6}\right)\left(x+\frac{x^{3}}{3}-\frac{2 x^{5}}{15}\right) \\
= & \frac{x^{5}}{15}\left(5+2,476 x^{2}+708 x^{4}-288 x^{6}\right)>0
\end{aligned}
$$

for $x \in(0,1)$.

Therefore, inequality (2.22) follows from (2.24), (2.25) and (2.29), and inequality (2.23) follows from (2.24), (2.25) and (2.30).

\section{Lemma 2.5 Let}

$$
\Upsilon(x)=\frac{1}{x\left(1+x^{2}\right)}-\frac{1}{\sqrt{1-x^{2}} \arcsin (x)} .
$$

Then the inequality

$$
\Upsilon(x)>-\frac{4 x}{3}+\frac{34 x^{3}}{45}-\frac{3 x^{5}}{2}
$$


holds for $x \in(0,0.7)$, and

$$
\Upsilon(x)<-\frac{4 x}{3}+\frac{34 x^{3}}{45}-\frac{8 x^{5}}{9}
$$

holds for $x \in(0,1)$.

Proof Let

$$
\begin{aligned}
\epsilon_{1}(x):= & x\left(1+x^{2}\right) \sqrt{1-x^{2}} \arcsin (x)\left[\Upsilon(x)+\left(\frac{4 x}{3}-\frac{34 x^{3}}{45}+\frac{3 x^{5}}{2}\right)\right] \\
= & \sqrt{1-x^{2}} \arcsin (x)-x\left(1+x^{2}\right) \\
& +x\left(1+x^{2}\right) \sqrt{1-x^{2}} \arcsin (x)\left(\frac{4 x}{3}-\frac{34 x^{3}}{45}+\frac{3 x^{5}}{2}\right)
\end{aligned}
$$

and

$$
\begin{aligned}
\epsilon_{2}(x):= & x\left(1+x^{2}\right) \sqrt{1-x^{2}} \arcsin (x)\left[\Upsilon(x)+\left(\frac{4 x}{3}-\frac{34 x^{3}}{45}+\frac{8 x^{5}}{9}\right)\right] \\
= & \sqrt{1-x^{2}} \arcsin (x)-x\left(1+x^{2}\right) \\
& +x\left(1+x^{2}\right) \sqrt{1-x^{2}} \arcsin (x)\left(\frac{4 x}{3}-\frac{34 x^{3}}{45}+\frac{8 x^{5}}{9}\right) .
\end{aligned}
$$

An easy calculation gives rise to

$$
\begin{aligned}
& \epsilon_{1}(0)=\epsilon_{2}(0)=0, \\
& \epsilon_{1}^{\prime}(x)=\frac{x}{90\left(1-x^{2}\right)} \epsilon_{1}^{*}(x), \quad \epsilon_{2}^{\prime}(x)=\frac{x}{45\left(1-x^{2}\right)} \epsilon_{2}^{*}(x),
\end{aligned}
$$

where

$$
\begin{aligned}
\epsilon_{1}^{*}(x)= & -x\left(150-202 x^{2}-15 x^{4}-68 x^{6}+135 x^{8}\right) \\
& +\left(150-152 x^{2}+142 x^{4}+611 x^{6}-1,215 x^{8}\right) \sqrt{1-x^{2}} \arcsin (x), \\
\epsilon_{2}^{*}(x)= & -x\left(1-x^{2}\right)\left(75-26 x^{2}-6 x^{4}-40 x^{6}\right) \\
& +\left(75-76 x^{2}-94 x^{4}+278 x^{6}-360 x^{8}\right) \sqrt{1-x^{2}} \arcsin (x) .
\end{aligned}
$$

Note that

$$
\begin{aligned}
& 150-152 x^{2}+142 x^{4}+611 x^{6}-1,215 x^{8} \\
& \quad>150-152 \times(0.7)^{2}-1,215 \times(0.7)^{8}=5.477 \ldots>0
\end{aligned}
$$

for $x \in(0,0.7)$. 
It follows from (2.10), (2.37) and (2.39) that

$$
\begin{aligned}
\epsilon_{1}^{*}(x)> & -x\left(150-202 x^{2}-15 x^{4}-68 x^{6}+135 x^{8}\right) \\
& +\left(150-152 x^{2}+142 x^{4}+611 x^{6}-1,215 x^{8}\right)\left(x-\frac{x^{3}}{3}-\frac{x^{5}}{3}\right) \\
= & \frac{x^{5}}{3}\left[\frac{1,183}{4}+709\left(\frac{1}{4}-x^{4}\right)+2,047 x^{2}\left(1-2 x^{2}\right)+604 x^{6}+1,215 x^{8}\right]>0
\end{aligned}
$$

for $x \in(0,0.7)$.

We claim that

$$
\epsilon_{2}^{*}(x)<0
$$

for $x \in(0,1)$. Indeed, let $q(x)=75-76 x^{2}-94 x^{4}+278 x^{6}-360 x^{8}$, then $q(0.8009)=$ $0.000171 \ldots, q(0.80091)=-0.00356 \ldots$ and

$$
q^{\prime}(x)=-4 x\left[38+\frac{10,759 x^{2}}{320}+720 x^{2}\left(x^{2}-\frac{139}{480}\right)\right]<0
$$

for $x \in(0,1)$. Therefore, there exists unique $x_{0}=0.80090 \ldots \in(0,1)$ such that $q(x)>0$ for $x \in\left(0, x_{0}\right)$ and $q(x) \leq 0$ for $\left[x_{0}, 1\right)$. This in conjunction with (2.11) and (2.38) leads to

$$
\begin{aligned}
\epsilon_{2}^{*}(x)< & -x\left(1-x^{2}\right)\left(75-26 x^{2}-6 x^{4}-40 x^{6}\right) \\
& +\left(75-76 x^{2}-94 x^{4}+278 x^{6}-360 x^{8}\right)\left(x-\frac{x^{3}}{3}-\frac{2 x^{5}}{15}\right) \\
= & -\frac{2 x^{5}}{15}\left[\frac{1,897,305,741}{27,436,644}+2,619\left(x^{2}-\frac{2,651}{5,238}\right)^{2}+2 x^{4}\left(1-x^{2}\right)\left(491+180 x^{2}\right)\right]<0
\end{aligned}
$$

for $x \in\left(0, x_{0}\right)$ and $\epsilon_{2}^{*}(x) \leq-x\left(1-x^{2}\right)\left(75-26 x^{2}-6 x^{4}-40 x^{6}\right)<0$ for $x \in\left[x_{0}, 1\right)$.

Therefore, inequality (2.31) follows from (2.33), (2.35), (2.36) and (2.40), and inequality (2.32) follows from (2.33)-(2.36) and (2.41).

\section{Lemma 2.6 Let}

$$
\mu(x)=\frac{1+3 x^{2}}{\left(x+x^{3}\right)^{2}}-\frac{1}{\left(1+x^{2}\right)\left[\sinh ^{-1}(x)\right]^{2}}-\frac{x}{\left(1+x^{2}\right)^{3 / 2} \sinh ^{-1}(x)} .
$$

Then $\mu(x)<0.2$ for $x \in[0.7,1)$.

Proof Let

$$
\mu_{1}(x)=\frac{1}{x^{2}}-\frac{1}{\left[\sinh ^{-1}(x)\right]^{2}}, \quad \mu_{2}(x)=\frac{2}{\sqrt{1+x^{2}}}-\frac{x}{\sinh ^{-1}(x)}
$$

Then

$$
\mu(x)=\frac{\mu_{1}(x)}{1+x^{2}}+\frac{\mu_{2}(x)}{\left(1+x^{2}\right)^{3 / 2}} .
$$


Lemma 2.2 together with $x>\sinh ^{-1}(x)$ gives $\mu_{1}(x)<0$ and

$$
\mu_{1}^{\prime}(x)=\frac{2}{x^{3}\left[\sinh ^{-1}(x)\right]^{3}}\left[\frac{x^{3}}{\sqrt{1+x^{2}}}-\left(\sinh ^{-1}(x)\right)^{3}\right]>0
$$

for $x \in(0,1)$. This in turn implies that

$$
\left[\frac{\mu_{1}(x)}{1+x^{2}}\right]^{\prime}=\frac{\mu_{1}^{\prime}(x)\left(1+x^{2}\right)-2 x \mu_{1}(x)}{\left(1+x^{2}\right)^{2}}>0
$$

for $x \in(0,1)$.

On the other hand, from the expression of $\mu_{2}(x)$, we get

$$
\begin{aligned}
& \mu_{2}(1)=0.2796 \ldots>0, \\
& \mu_{2}^{\prime}(x)=-\frac{2 x}{\left(1+x^{2}\right)^{3 / 2}}+\frac{\mu_{2}^{*}(x)}{\left[\sinh ^{-1}(x)\right]^{2}},
\end{aligned}
$$

where

$$
\begin{aligned}
& \mu_{2}^{*}(x)=\frac{x}{\sqrt{1+x^{2}}}-\sinh ^{-1}(x), \\
& \mu_{2}^{*}(0)=0, \\
& \mu_{2}^{* \prime}(x)=-\frac{x^{2}}{\left(1+x^{2}\right)^{3 / 2}}<0
\end{aligned}
$$

for $x \in(0,1)$.

From (2.44)-(2.48) we clearly see that $\mu_{2}^{\prime}(x)<0$ and $\mu_{2}(x)>0$ for $x \in(0,1)$. This in turn implies that

$$
\left[\frac{\mu_{2}(x)}{\left(1+x^{2}\right)^{3 / 2}}\right]^{\prime}=\frac{\mu_{2}^{\prime}(x)\left(1+x^{2}\right)^{3 / 2}-3 x \sqrt{1+x^{2}} \mu_{2}(x)}{\left(1+x^{2}\right)^{3}}<0
$$

for $x \in(0,1)$.

Equation (2.42) and inequalities (2.43) and (2.49) lead to the conclusion that

$$
\mu(x) \leq \frac{\mu_{1}(1)}{2}+\frac{\mu_{2}(0.7)}{\left[1+(0.7)^{2}\right]^{3 / 2}}=0.167 \ldots<0.2
$$

for $x \in[0.7,1)$.

Lemma 2.7 Let

$$
v(x)=-\frac{1+3 x^{2}}{\left(x+x^{3}\right)^{2}}+\frac{1}{\left(1-x^{2}\right) \arcsin ^{2}(x)}-\frac{x}{\left(1-x^{2}\right)^{3 / 2} \arcsin (x)} .
$$

Then $v(x)<-1.48$ for $x \in[0.7,1)$.

Proof Differentiating $v(x)$ yields

$$
v^{\prime}(x)=\frac{\left(x+x^{3}\right)^{3} \arcsin (x) v_{1}(x)+\left(1-x^{2}\right) v_{2}(x)}{x^{3}\left(1-x^{2}\right)^{5 / 2}\left(1+x^{2}\right)^{3} \arcsin ^{3}(x)},
$$


where

$$
\begin{aligned}
& \nu_{1}(x)=3 x \sqrt{1-x^{2}}-\left(1+2 x^{2}\right) \arcsin (x), \\
& \nu_{2}(x)=2\left(1+3 x^{2}+6 x^{4}\right)\left[\sqrt{1-x^{2}} \arcsin (x)\right]^{3}-2\left(x+x^{3}\right)^{3} .
\end{aligned}
$$

Equation (2.51) leads to

$$
\begin{aligned}
& v_{1}(0.7)=-0.03558 \ldots, \\
& v_{1}^{\prime}(x)=\frac{2-8 x^{2}-4 x \sqrt{1-x^{2}} \arcsin (x)}{\sqrt{1-x^{2}}}<0
\end{aligned}
$$

for $x \in[0.7,1)$.

Therefore,

$$
v_{1}(x)<0
$$

for $x \in[0.7,1)$ follows from $(2.53)$ and $(2.54)$.

It follows from (2.52) and (2.11) that

$$
\begin{aligned}
v_{2}(x) & <2\left(1+3 x^{2}+6 x^{4}\right)\left(x-\frac{x^{3}}{3}\right)^{3}-2\left(x+x^{3}\right)^{3} \\
& =-\frac{2 x^{5}}{27}\left(27-9 x^{2}+163 x^{4}-51 x^{6}+6 x^{8}\right)<0
\end{aligned}
$$

for $x \in[0.7,1)$.

Equation (2.50) together with inequalities (2.55) and (2.56) leads to the conclusion that $v(x)$ is strictly decreasing on $[0.7,1)$. This in turn implies that

$$
v(x) \leq v(0.7)=-1.48798 \ldots<-1.48
$$

for $x \in[0.7,1)$.

Lemma 2.8 Let $\lambda_{0}=[2 \log (\log (1+\sqrt{2}))+\log 2] /[2 \log \pi-\log 2]=0.2760 \ldots$, and $\Theta(x)=$ $\Phi(x)+\lambda_{0} \Upsilon(x)$, where $\Phi(x)$ and $\Upsilon(x)$ are defined as in Lemmas 2.4 and 2.5, respectively. Then the function $\Theta(x)$ is strictly decreasing on $[0.7,1)$.

Proof Let $\mu(x)$ and $v(x)$ be defined as in Lemmas 2.6 and 2.7, respectively. Then differentiating $\Theta(x)$ yields

$$
\Theta^{\prime}(x)=\Phi^{\prime}(x)+\lambda_{0} \Upsilon^{\prime}(x)=\mu(x)+\lambda_{0} v(x)<0.2-1.48 \lambda_{0}=-0.208 \ldots<0
$$

for $x \in[0.7,1)$. This in turn implies that $\Theta(x)$ is strictly decreasing on $[0.7,1)$.

\section{Main result}

Theorem 3.1 The double inequality

$$
P^{\alpha}(a, b) Q^{1-\alpha}(a, b)<M(a, b)<P^{\beta}(a, b) Q^{1-\beta}(a, b)
$$


holds for all $a, b>0$ with $a \neq b$ if and only if $\alpha \geq 1 / 2$ and $\beta \leq[2 \log (\log (1+\sqrt{2}))+$ $\log 2] /[2 \log \pi-\log 2]=0.2760 \ldots$.

Proof Since $P(a, b), M(a, b)$ and $Q(a, b)$ are symmetric and homogeneous of degree 1, without loss of generality, we assume that $a>b$. Let $p \in(0,1), \lambda_{0}=[2 \log (\log (1+\sqrt{2}))+$ $\log 2] /[2 \log \pi-\log 2]$ and $x=(a-b) /(a+b)$. Then $x \in(0,1)$,

$$
\begin{aligned}
& \frac{P(a, b)}{A(a, b)}=\frac{x}{\arcsin (x)}, \quad \frac{M(a, b)}{A(a, b)}=\frac{x}{\sinh ^{-1}(x)}, \quad \frac{Q(a, b)}{A(a, b)}=\sqrt{1+x^{2}}, \\
& \frac{\log [Q(a, b)]-\log [M(a, b)]}{\log [Q(a, b)]-\log [P(a, b)]}=\frac{\log \left(1+x^{2}\right)-2 \log x+2 \log \left[\sinh ^{-1}(x)\right]}{\log \left(1+x^{2}\right)-2 \log x+2 \log [\arcsin (x)]}
\end{aligned}
$$

and

$$
\begin{aligned}
& \lim _{x \rightarrow 0^{+}} \frac{\log \left(1+x^{2}\right)-2 \log x+2 \log \left[\sinh ^{-1}(x)\right]}{\log \left(1+x^{2}\right)-2 \log x+2 \log [\arcsin (x)]}=\frac{1}{2}, \\
& \lim _{x \rightarrow 1^{-}} \frac{\log \left(1+x^{2}\right)-2 \log x+2 \log \left[\sinh ^{-1}(x)\right]}{\log \left(1+x^{2}\right)-2 \log x+2 \log [\arcsin (x)]}=\lambda_{0} .
\end{aligned}
$$

The difference between the convex combination of $\log [P(a, b)], \log [Q(a, b)]$ and $\log [M(a$, b)] is given by

$$
\begin{aligned}
& p \log [P(a, b)]+(1-p) \log [Q(a, b)]-\log [M(a, b)] \\
& \quad=p \log \left[\frac{x}{\arcsin (x)}\right]+\frac{1-p}{2} \log \left(1+x^{2}\right)-\log \left[\frac{x}{\sinh ^{-1}(x)}\right]:=D_{p}(x) .
\end{aligned}
$$

Equation (3.4) leads to

$$
\begin{aligned}
& D_{p}\left(0^{+}\right)=0, \quad D_{p}\left(1^{-}\right)=\log [\sqrt{2} \log (1+\sqrt{2})]-p \log \left(\frac{\pi}{\sqrt{2}}\right), \quad D_{\lambda_{0}}\left(1^{-}\right)=0, \\
& D_{p}^{\prime}(x)=-\frac{p}{\sqrt{1-x^{2}} \arcsin (x)}-\frac{(1-p)}{x\left(1+x^{2}\right)}+\frac{1}{\sqrt{1+x^{2}} \sinh ^{-1}(x)}=\Phi(x)+p \Upsilon(x),
\end{aligned}
$$

where $\Phi(x)$ and $\Upsilon(x)$ are defined as in Lemmas 2.4 and 2.5, respectively.

From Lemmas 2.4 and 2.5 , we clearly see that

$$
\begin{aligned}
D_{1 / 2}^{\prime}(x) & =\Phi(x)+\frac{1}{2} \Upsilon(x) \\
& <\frac{2 x}{3}-\frac{34 x^{3}}{45}+\frac{4 x^{5}}{5}-\frac{1}{2}\left(\frac{4 x}{3}-\frac{34 x^{3}}{45}+\frac{8 x^{5}}{9}\right) \\
& =-\frac{16 x^{3}}{45}\left(\frac{17}{16}-x^{2}\right)<0
\end{aligned}
$$

for $x \in(0,1)$, and

$$
\begin{aligned}
D_{\lambda_{0}}^{\prime}(x) & =\Phi(x)+\lambda_{0} \Upsilon(x) \\
& >\frac{2 x}{3}-\frac{34 x^{3}}{45}+\frac{754 x^{5}}{945}-x^{7}-\lambda_{0}\left(\frac{4 x}{3}-\frac{34 x^{3}}{45}+\frac{3 x^{5}}{2}\right)
\end{aligned}
$$




$$
\begin{aligned}
& =x\left[\frac{2\left(1-2 \lambda_{0}\right)}{3}-\frac{34\left(1-\lambda_{0}\right)}{45} x^{2}+\left(\frac{754}{945}-\frac{3 \lambda_{0}}{2}\right) x^{4}-x^{6}\right] \\
& :=x F_{\lambda_{0}}(x)>0
\end{aligned}
$$

for $x \in(0,0.7)$.

Note that

$$
F_{\lambda_{0}}(0)=2\left(1-2 \lambda_{0}\right) / 3>0, \quad F_{\lambda_{0}}(0.7)=0.00513 \ldots>0
$$

and

$$
\begin{aligned}
F_{\lambda_{0}}^{\prime \prime}(x) & =-30\left[\left(x^{2}-\frac{1,508-2,835 \lambda_{0}}{9,450}\right)^{2}+\frac{2,224,136+4,052,160 \lambda_{0}-8,037,225 \lambda_{0}^{2}}{89,302,500}\right] \\
& <0
\end{aligned}
$$

for $x \in(0,0.7)$.

Inequalities (3.8)-(3.10) lead to the conclusion that

$$
D_{\lambda_{0}}^{\prime}(x)>0
$$

for $x \in(0,0.7)$.

It follows from Lemma 2.8 and $(3.6)$ that $D_{\lambda_{0}}^{\prime}(x)$ is strictly decreasing in $[0.7,1)$. Then from (3.11) and $D_{\lambda_{0}}^{\prime}(0.7)=0.0626 \ldots$ together with $D_{\lambda_{0}}^{\prime}\left(1^{-}\right)=-\infty$, we know that there exists $x^{*} \in(0.7,1)$ such that $D_{\lambda_{0}}(x)$ is strictly increasing on $\left(0, x^{*}\right]$ and strictly decreasing on $\left[x^{*}, 1\right)$. This in conjunction with $(3.5)$ implies that

$$
D_{\lambda_{0}}(x)>0
$$

for $x \in(0,1)$.

Equations (3.4), (3.5), (3.7) and (3.12) lead to the conclusion that

$$
M(a, b)<P^{\lambda_{0}}(a, b) Q^{1-\lambda_{0}}(a, b)
$$

and

$$
M(a, b)>P^{1 / 2}(a, b) Q^{1 / 2}(a, b)
$$

Therefore, Theorem 3.1 follows from (3.13) and (3.14) together with the following statements:

- If $\alpha<1 / 2$, then (3.1) and (3.2) imply that there exists $\delta_{1} \in(0,1)$ such that $M(a, b)<P^{\alpha}(a, b) Q^{1-\alpha}(a, b)$ for all $a, b>0$ with $(a-b) /(a+b) \in\left(0, \delta_{1}\right)$.

- If $\beta>\lambda_{0}$, then (3.1) and (3.3) imply that there exists $\delta_{2} \in(0,1)$ such that $M(a, b)>P^{\beta}(a, b) Q^{1-\beta}(a, b)$ for all $a, b>0$ with $(a-b) /(a+b) \in\left(1-\delta_{2}, 1\right)$. 
Authors' contributions

W-MG provided the main idea and carried out the proof of Theorem 3.1. X-HS carried out the proof of Lemmas 2.1-2.5.

Y-MC carried out the proof of Lemmas 2.6-2.8. All authors read and approved the final manuscript.

\section{Author details}

'School of Mathematics and Computation Science, Hunan City University, Yiyang, 413000, China. ${ }^{2}$ College of Nursing, Huzhou Teachers College, Huzhou, 313000, China.

\section{Acknowledgements}

This research was supported by the Natural Science Foundation of China under Grants 11071069 and 11171307, and the Natural Science Foundation of Zhejiang Province under Grants LY13H070004 and LY13A010004.

Received: 12 July 2013 Accepted: 18 October 2013 Published: 22 Nov 2013

\section{References}

1. Seiffert, H-J: Problem 887. Nieuw Arch. Wiskd. 11(2), 176 (1993)

2. Neuman, E, Sándor, J: On the Schwab-Borchardt mean. Math. Pannon. 14(2), 253-266 (2003)

3. Chu, Y-M, Hou, S-W, Shen, Z-H: Sharp bounds for Seiffert mean in terms of root mean square. J. Inequal. Appl. 2012, $11(2012)$

4. Chu, Y-M, Wang, M-K: Refinements of the inequalities between Neuman-Sándor, arithmetic, contra-harmonic and quadratic means. arXiv:1209.2920v1 [math.CA]

5. Chu, Y-M, Wang, M-K, Qiu, S-L: Optimal combinations bounds of root-square and arithmetic means for Toader mean. Proc. Indian Acad. Sci. Math. Sci. 122(1), 41-51 (2012)

6. Chu, Y-M, Wang, M-K, Wang, Z-K: Best possible inequalities among harmonic, geometric, logarithmic and Seiffert means. Math. Inequal. Appl. 15(2), 415-422 (2012)

7. Hästö, PA: A monotonicity property of ratios of symmetric homogeneous means. JIPAM. J. Inequal. Pure Appl. Math. 3(5), Article ID 71 (2002)

8. Hästö, PA: Optimal inequalities between Seiffert's mean and power mean. Math. Inequal. Appl. 7(1), 47-53 (2004)

9. Gao, S-Q: Inequalities for the Seiffert's means in terms of the identric mean. J. Math. Sci. Adv. Appl. 10(1-2), 23-31 (2011)

10. Gong, W-M, Song, Y-Q, Wang, M-K, Chu, Y-M: A sharp double inequality between Seiffert, arithmetic, and geometric means. Abstr. Appl. Anal. 2012, Article ID 684834 (2012)

11. Jiang, W-D: Some sharp inequalities involving reciprocals of the Seiffert and other means. J. Math. Inequal. 6(4), 593-599 (2012)

12. Liu, H, Meng, J-X: The optimal convex combination bounds for Seiffert's mean. J. Inequal. Appl. 2011, Article ID $686834(2011)$

13. Neuman, E: A note on a certain bivariate mean. J. Math. Inequal. 6(4), 637-643 (2012)

14. Neuman, E, Sándor, J: On certain means of two arguments and their extension. Int. J. Math. Math. Sci. 16, 981-993 (2003)

15. Neuman, E, Sándor, J: On the Schwab-Borchardt mean II. Math. Pannon. 17(1), 49-59 (2006)

16. Li, Y-M, Long, B-Y, Chu, Y-M: Sharp bounds for the Neuman-Sándor mean in terms of generalized logarithmic mean. J. Math. Inequal. 6(4), 567-577 (2012)

17. Jiang, W-D, Qi, F: Sharp bounds for Neuman-Sándor mean in terms of the root-mean-square. arXiv:1301.3267v1 [math.CA]

18. Jiang, W-D, Qi, F: Sharp bounds in terms of a two-parameter family of means for Neuman-Sándor mean. Preprint

10.1186/1029-242X-2013-552

Cite this article as: Gong et al.: Optimal bounds for the Neuman-Sándor mean in terms of the first Seiffert and quadratic means. Journal of Inequalities and Applications 2013, 2013:552

\section{Submit your manuscript to a SpringerOpen ${ }^{\circ}$ journal and benefit from:}

- Convenient online submission

Rigorous peer review

- Immediate publication on acceptance

- Open access: articles freely available online

- High visibility within the field

- Retaining the copyright to your article 